\title{
Melanoma of the vulva: A retrospective study of 46 cases from Queensland*
}

\author{
Ian S. C. Jones ${ }^{\#}$, Alex Crandon, Karen Sanday \\ Queensland Centre for Gynaecological Cancer, Royal Brisbane and Women’s Hospital, Brisbane, Australia \\ Email: ${ }^{\#}$ Ian_Jones@health.qld.gov.au
}

Received 27 March 2012; revised 25 April 2012; accepted 7 May 2012

\begin{abstract}
Objective: To review the records of cases of vulvar melanoma referred to the Queensland Centre for Gynaecological Cancer (QCGC) between 1986 and 2009 recognising the high incidence of cutaneous melanoma in Queensland. Methods: Vulvar melanoma case data from QCGC were reviewed and analysed using the computer software Statistical Package for the Social Sciences (SPSS) 11.0. Results: Of the 46 cases 22 died of their disease, nine died of other diseases, ten are still alive and five lost to follow up. The mean age at diagnosis was 65.5 years for nodular melanoma and 69 years for superficial spreading melanoma. All cases were Caucasian. Time from on-set of symptoms to diagnosis averaged 3.2 months. In all 46 cases diagnosis was confirmed histologically. Presenting symptoms included lumps, itch, stinging, bleeding and pain. The most common presenting complaint was a lump. The initial treatment was surgical without a trend to a less radical approach to management. Conclusions: Vulvar melanoma prognosis remains guarded. Increased tumor size, depth of invasion, mitotic rate, groin lymph node positivity and status of disease at the margins of surgical specimens reliably equate to long term outcome. The incidence of vulvar melanoma was not increased in Queensland.
\end{abstract}

Keywords: Vulvar Melanoma; Diagnosis; Management; Outcome; Follow-Up

\section{INTRODUCTION}

Queensland, Australia has the world's highest incidence rates of invasive cutaneous melanoma (50 per 100,000 for women and 75 per 100,000 for men) [1,2] with the vulva being an uncommon site of melanoma $(<1 \%)$ [3]. However vulvar melanoma is the second most common

\footnotetext{
*Conflict of interest: the authors declare that there are no conflicts of interest.

${ }^{\#}$ Corresponding author.
}

vulvar malignancy [3] with an incidence that has been variously reported as between $1 \%-10 \%$ of vulvar cancers $[3,4]$. The cell origin is the melanocyte with approximately $10 \%$ arising in pre existing vulvar naevi [5].

The aim of the study was to review the clinical features, diagnosis, management and outcomes from the patient records of cases of vulvar melanoma held in the Queensland Centre for Gynaecological Cancer (QCGC) data base and determine if the surgical management and patient outcome altered in any way over the study period.

\section{MATERIALS AND METHODS}

Case records, including pathology reports from the state wide QCGC data base for patients referred for registration and management of malignant melanoma of the vulva between 1986 and 2009 were reviewed and analysed using the computer software Statistical Package for the Social Sciences (SPSS) 11.0. Where data was found to be incomplete attempts were made to collect this information by reviewing patient case notes and pathology department records.

Ethics approval for the review of case records was obtained from the Clinical Research Ethics Committee of the Royal Brisbane and Women's Hospital.

\section{RESULTS}

Forty-six cases of vulvar malignant melanoma were registered with QCGC and all were Caucasian. The number of cases registered each year ranged from zero to five. The incidence of vulvar melanoma as a percentage of the number of vulvar cancer presenting each year ranged between zero to $9.8 \%$. Of the 46 patients 22 have died of their disease, nine have died of other diseases, ten are still alive and five were lost to follow up. The five cases who presented early in the series were lost to follow up after an average of 127 months (range 61 - 215 months) of follow up. The mean age at diagnosis was 65.5 years (range 25 - 93) for the 40 cases of nodular melanoma and 69 years (range 52 - 77) for the five cases of superficial spreading melanoma. The one case of amelanotic mela- 
noma was aged 41 years at diagnosis. Age versus outcome data is presented in Table $\mathbf{1}$.

Time from onset of symptoms to diagnosis averaged 3.2 months (3.3 months for nodular melanoma, 1.6 months for superficial spreading melanoma and 6 months for the single case of amelanotic melanoma, with an overall range of nil to 24 months). In all 46 cases the diagnosis was confirmed by histology (40 nodular, 5 superficial spreading and 1 amelanotic). Following further attempts at gaining information histology and immunohistochemistry reports (rather than report summaries) were available for review in 35 cases. This accounts for the differences in some table totals. Presenting symptoms included lumps, itch, stinging, bleeding, pain and various combinations of these symptoms. The most common presenting complaints were a lump (in 38\% of cases) and vulvar itch (in $31 \%$ of cases). The location of the lesion on the vulva where this information was documented in the QCGC data base was 11 on the right, 10 on the left of the vulva and 8 on the clitoris.

Treatment regimes were adapted to the patient's overall condition by the treating surgeon rather than following a set protocol. Surgical procedures included wide local excision with or without radiotherapy, hemi vulvectomy with or without groin node dissection, vulvectomy, radical vulvectomy with groin dissection, radical vulvectomy with groin dissection and pelvic lymphadenectomy. In all cases the initial treatment was surgical. Local radiation treatment was used in 12 cases (two in conjunction with initial surgery and ten in subsequent treatments) and one case underwent chemotherapy but this treatment regime was not recorded in the QCGC data base.

Seven of the 31 cases who under went groin or sentinel node biopsy are alive, with six of the seven having negative nodes (Table 2). Sentinel node biopsy was performed in 18 cases (7 positive, 11 negative) from 1999 onward (Table 3). Surgical treatment trends between 1990-
1999 and 2000-2009 did not change significantly despite the proportion of more radical treatments increasing from 69 to 88 per cent (z score $=1.175=$ not significant) between these two decades. The tumor size $>10 \mathrm{~mm}$ (Table 4 , Dead of disease $=13 / 16=81 \%$, z score $=4.328$, significant at 95\%); depth of invasion $>1 \mathrm{~mm}$ (Table 5, Dead of disease $=12 / 18=67 \%$, z score $=5.476=$ significant at $95 \%$ ); an increased mitotic rate variously reported as the study progressed as numerous, high or as found later in the study as the number per $\mathrm{mm}^{3}$ (Table 6), and a reduced distance of tumor to the excised skin margin (Table 7) did aversely affect outcome. Positive tumor specimen margins occurred in 5 (14\%) cases with 31 ( $86 \%$, z score $=5.892=$ significant difference) having clear margins. Note this excluded the 10 cases where there was no record for the state of the specimen margins in either the QCGC data or the pathology report. However, tumor ulceration did not affect outcome. Negative groin lymph nodes increased the survival time with six of these 17 being currently alive (Table 8). The average survival time when the groin lymph nodes are negative was 66.6 months but fell to 13.7 months if positive.

Table 1. Age versus outcome.

\begin{tabular}{cccccc}
\hline Age & Alive & Dead of disease & Dead other & Lost to FU & Total \\
\hline$<40$ & 0 & 0 & 0 & 1 & 1 \\
$40-49$ & 6 & 1 & 0 & 2 & 9 \\
$50-59$ & 1 & 5 & 0 & 1 & 7 \\
$60-69$ & 1 & 5 & 0 & 0 & 6 \\
$70-79$ & 0 & 8 & 6 & 0 & 14 \\
$80-89$ & 1 & 3 & 2 & 1 & 7 \\
$90+$ & 1 & 0 & 1 & 0 & 2 \\
Total & 10 & 22 & 9 & 5 & 46 \\
\hline
\end{tabular}

Table 2. Patient health status versus regional lymph node status.

\begin{tabular}{|c|c|c|c|c|c|}
\hline \multirow{2}{*}{ Lymph node status } & \multicolumn{5}{|c|}{ STATUS } \\
\hline & Alive & Dead of disease & Dead other disease & Lost to FU & Total \\
\hline Sampled no histology available & 0 & 3 & 1 & 0 & 4 \\
\hline Sample negative & 6 & 4 & 3 & 4 & 17 \\
\hline Sample positive & 1 & 8 & 1 & 0 & 10 \\
\hline Not sampled & 3 & 4 & 4 & 0 & 11 \\
\hline Not recorded & 0 & 1 & 0 & 1 & 2 \\
\hline Recurrence & 0 & 2 & 0 & 0 & 2 \\
\hline Total & 10 & 22 & 9 & 5 & 46 \\
\hline
\end{tabular}


Table 3. Sentinel node status versus outcome.

\begin{tabular}{|c|c|c|c|c|c|}
\hline \multirow{2}{*}{ Sentinel node } & \multicolumn{5}{|c|}{ STATUS } \\
\hline & Alive & Dead of disease & Dead other disease & Lost to FU & Total \\
\hline Positive & 1 & 5 & 1 & 0 & 7 \\
\hline Negative & 6 & 2 & 2 & 1 & 11 \\
\hline Not done & 3 & 4 & 3 & 0 & 10 \\
\hline Recurrence & 0 & 2 & 0 & 0 & 2 \\
\hline Unknown & 0 & 9 & 3 & 4 & 16 \\
\hline Total & 10 & 22 & 9 & 5 & 46 \\
\hline
\end{tabular}

Table 4. Size of melanoma versus outcome status.

\begin{tabular}{|c|c|c|c|c|c|}
\hline \multirow{2}{*}{ Tumor size mm } & \multicolumn{5}{|c|}{ STATUS } \\
\hline & Alive & Dead of disease & Dead other disease & Lost to FU & Total \\
\hline 1 & 1 & & & & 1 \\
\hline 3 & 2 & & & & 2 \\
\hline 4 & & 1 & 1 & & 2 \\
\hline 6 & 1 & & 1 & & 2 \\
\hline 7 & & 1 & & & 1 \\
\hline 9 & 1 & & & & 1 \\
\hline 10 & & 1 & & & 1 \\
\hline 11 & & 1 & 1 & & 2 \\
\hline 15 & 1 & 2 & & & 3 \\
\hline 17 & & & 1 & & 1 \\
\hline 18 & & 1 & & & 1 \\
\hline 19 & & 1 & & & 1 \\
\hline 20 & & 1 & & & 1 \\
\hline 21 & & 1 & 1 & & 2 \\
\hline 25 & & 1 & 1 & & 2 \\
\hline 30 & 1 & 2 & & & 3 \\
\hline 32 & & & & 1 & 1 \\
\hline 40 & 1 & & & & 1 \\
\hline 41 & & 1 & & & 1 \\
\hline 45 & & 1 & & & 1 \\
\hline 54 & & & 1 & & 1 \\
\hline 55 & & 1 & & & 1 \\
\hline Total & 8 & 16 & 7 & 1 & 32 \\
\hline
\end{tabular}


Table 5. Depth of melanoma lesion.

\begin{tabular}{|c|c|c|c|c|c|}
\hline \multirow{2}{*}{ Depth mm } & \multicolumn{5}{|c|}{ STATUS } \\
\hline & Alive & Dead of disease & Dead of other disease & Lost to FU & Total \\
\hline 0.00 & 2 & & & & 2 \\
\hline 0.42 & 1 & & & & 1 \\
\hline 0.50 & & 1 & & & 1 \\
\hline 0.55 & & 1 & & & 1 \\
\hline 0.59 & 1 & & & & 1 \\
\hline 0.75 & 1 & & & & 1 \\
\hline 1.20 & 1 & & & & 1 \\
\hline 1.40 & & 1 & & & 1 \\
\hline 1.70 & 1 & & & & 1 \\
\hline 2.00 & & & & 1 & 1 \\
\hline 2.20 & & 1 & & & 1 \\
\hline 3.50 & & 1 & & & 1 \\
\hline 4.60 & & & 1 & & 1 \\
\hline 5.00 & 1 & 2 & & & 3 \\
\hline 5.20 & & 1 & & & 1 \\
\hline 6.00 & & 1 & & & 1 \\
\hline 7.00 & & 1 & & & 1 \\
\hline 8.00 & & 1 & & & 1 \\
\hline 9.00 & & 1 & & & 1 \\
\hline 20.00 & & 2 & & & 2 \\
\hline 30.00 & & & 1 & & 1 \\
\hline Total & 8 & 14 & 2 & 1 & 25 \\
\hline
\end{tabular}

In one case there was a suspicion that a vulvar melanoma had arisen in a pre-existing vulvar naevus. There were no documented cases with a family history of melanoma in non vulvar locations. However, nine of the 46 cases had another malignancy prior to the diagnosis of the vulvar melanoma. These nine cases included four with limb or trunk melanomas, one non-melanotic skin cancer, and one adenocarcinoma of the small intestine, one breast cancer, one adrenal cancer and one cancer where the site was not documented in the QCGC records.

\section{DISCUSSION}

As noted in the introduction melanoma of the vulva is the second most common vulvar malignancy with an incidence that has been variously reported as between $1 \%-10 \%$ of all vulvar cancers. In our series the incidence varied between zero and 9.8\% per year thus agree- ing with other reports. The mean age at diagnosis was 65.5 years (range 25 - 93 years) for nodular melanoma and 69 years (range 52 - 77 years) for superficial spreading melanoma. The mean age at presentation for vulvar melanoma was 6 - 10 years younger than some reports $[3,6]$ but not others $[7,8]$. This difference does not appear to be associated with country of origin nor year of presentation. Time from onset of symptoms to diagnosis averaged 3.2 months (range nil to 24 months). Presenting symptoms were similar to other reports [6] including lumps, itch, stinging, bleeding and pain with the most common presenting complaints being a lump (38\%) and itch (31\%). There was no identifiable "favoured" site on the vulva for malignant melanoma but as the QCGC records did not localize the lesion site for 17 of 46 cases this observation can not be considered reliable.

Vulvar biopsy was the final arbiter with differentiation 
Table 6. Mitotic rate versus outcome.

\begin{tabular}{ccccc}
\hline Mitoses & & & STATUS & \\
\cline { 2 - 4 } & Alive & Dead of disease & Dead other & 5 \\
\hline Numerous & 0 & 2 & 3 & 5 \\
High & 0 & 4 & 1 & 2 \\
1.00 & 1 & 1 & 0 & 1 \\
2.00 & 0 & 1 & 0 & 1 \\
3.00 & 1 & 0 & 1 \\
5.00 & 0 & 1 & 0 & 1 \\
8.00 & 0 & 1 & 0 & 1 \\
9.00 & 1 & 0 & 0 & 1 \\
10.00 & 0 & 0 & 1 & 1 \\
12.00 & 0 & 0 & 1 & 1 \\
24.00 & 0 & 1 & 0 & 0 \\
28.00 & 0 & 1 & 6 & 21 \\
Total & 3 & 12 & & 1 \\
\hline
\end{tabular}

Table 7. Specimen margin versus outcome.

\begin{tabular}{ccccc}
\hline Margin status & Outcome alive & Dead of disease & Dead of other disease & Lost to FU \\
\hline Clear & 3 & 8 & 2 & 1 \\
Tumor to margin & 0 & 4 & 3 & 0 \\
$1<6$ mm from skin margin & 2 & 3 & 0 & 0 \\
6 - 8 mm from skin margin & 3 & 1 & 0 & 0 \\
$15-22$ mm from skin margin & 2 & 0 & & \\
\hline
\end{tabular}

between melanoma, squamous cell carcinoma and Pagets Disease requiring special techniques [9]. The average survival time for those with positive groin lymph nodes was 13.7 months as compared with 66.6 months for those with negative nodes. Sentinel node biopsy was performed in the majority of cases from 1999 onwards. Prognostic factors from the 2009 AJCC Melanoma Staging and Classification report for cutaneous melanomas included localization, tumor thickness, mitotic rate and ulceration [10]. We found increased tumour size $>10 \mathrm{~mm}$, depth of invasion $>1 \mathrm{~mm}$, increased mitotic rate and a small distance of the tumor to the excised skin margin adversely affected the outcome for the patient but tumor ulceration did not adversely affect outcome.

Four of 36 cases (11\%) where the QCGC records were complete had previous non vulvar cutaneous melanomas and one had a vulval naevus but it is not known if this lesion progressed to a vulvar melanoma. Three of the four cases with non vulvar melanoma are alive but the other died of her vulvar melanoma. The vulvar naevus case also died of her vulvar melanoma.

All initial treatment was surgical. In Queensland, apart from the use of sentinel node biopsy rather than block dissection of groin lymph nodes, there was no significant trend to a less radical approach to the management of the vulvar lesion between the two decades. This finding is contrary to other reports $[11,12]$ who in the 1990's had favored wide local excision, especially for thin lesions. However, a more recent report [13] showed that the less radical approach to surgery for both vulvar melanoma and squamous carcinoma, although showing patient benefit with its decreased morbidity, unfortunately led to a higher local and regional recurrence rate. Similar warnings regarding the possible risk of higher local and regional recurrence when relying on sentinel lymph node biopsy have been raised by others [7]. Our review of 
Table 8. Survival time versus groin lymph node status versus outcome.

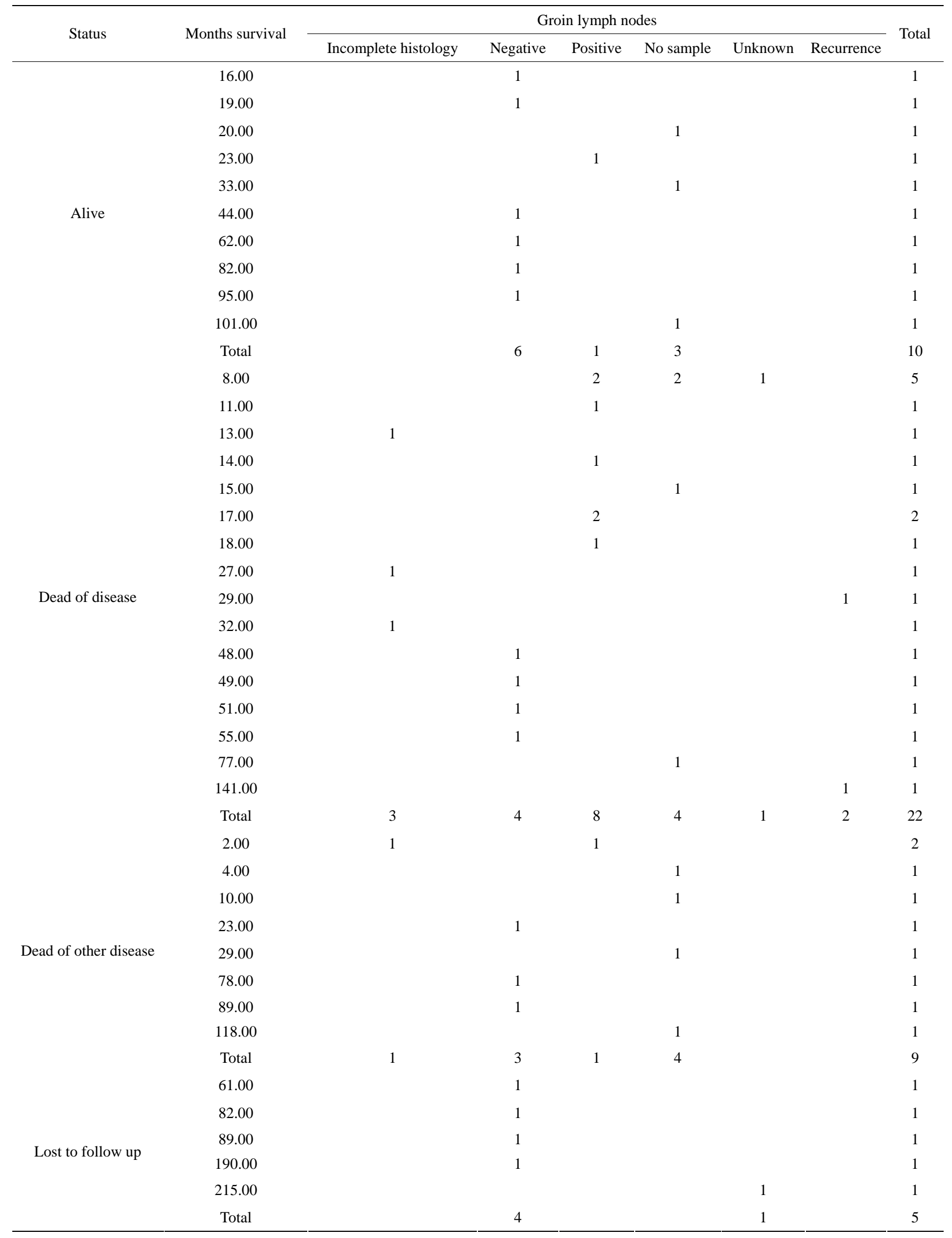


sentinel node biopsy in 18 cases (7 positive, 11 negative) found $1 / 7(14 \%)$ and $6 / 11(54.5 \%)$ cases currently remain alive and disease free ( $\mathrm{z}$ score $=0.748=$ no significant difference). Of the 11 negative sentinel node cases, three underwent less radical surgery and one underwent radical surgery but all four experienced recurrences. Of the remaining seven cases with no recurrence, four underwent radical surgery and three less radical surgery. These findings suggest the added need for close long term follow up when using the less radical surgical approach to treating vulvar melanoma.

In the current study the survival rate was $76 \%$ at one year, $52 \%$ at two years and $30 \%$ at five years. We agree with others that the five year $[14,15]$ or even 10 year [16] survival rate does not mean cure. The longest time to recurrence in our series was 11.7 years. The prognosis for vulvar melanoma must remain guarded despite some features of the disease having more favourable significance than others. Because of this uncertainty regarding possible cure we recommend careful long term follow up, irrespective of treatment modality, size and depth of the lesion, groin lymph node status or status of disease at the margins of surgical specimens (where neither positive nor negative findings reliably equate to long term outcome).

Despite Queensland having a very high incidence of cutaneous melanoma the incidence of vulvar melanoma is similar to the other studies referred to above. Of interest is the study of anorectal melanoma in Queensland [17] which concluded "The protection against cutaneous melanoma afforded by skin pigmentation is not apparent in the anal area. Anorectal melanoma is no more or less frequent in Queensland, even with the very high incidence rate of cutaneous melanoma. Sun exposure appears to be neither protective nor a risk factor". We consider these findings equally applicable to vulvar melanoma.

\section{ACKNOWLEDGEMENTS}

We gratefully acknowledge all those doctors who referred cases to the QCGC and the gynaecological oncologists who have updated QCGC records.

\section{REFERENCES}

[1] Incidence of cutaneous melanoma in Queensland 19822007.

http://www.cancerqld.org.au/f/QCSOL/View/Incidence_( Diagnosis)/Trends_Over_Time/Melanoma/By_Sex

[2] Coory, M., Baade, P., Aitken, J., Smithers, M., McLeod, G.R.C. and Ring, I. (2006) Trends for in situ and invasive melanoma in Queensland, Australia, 1982-2002. Cancer Causes and Control, 17, 21-27. doi:10.1007/s10552-005-3637-4

[3] Wechter, M.E., Gruber, S.B., Haefner, H.K., Lowe, L., Schwartz, J.L., Reynolds, K.R., Johnston, C.M. and John- son, T.M. (2004) Vulvar melanoma: A report of 20 cases and a review of the literature. Journal of the American Academy of Dermatology, 50, 554-562. doi:10.1016/j.jaad.2003.07.026

[4] Rollason, T.P. (1992) Malignant melanoma and related lesions of the lower female genital tract. In: Lowe, D. and H., Fox, Eds., Advances in Gynaecological Pathology, Churchill Livingstone, Edinburgh, 119-143.

[5] Curtin, J.P. and Morrow, C.P. (1992) Melanoma of the lower female genital tract. In: Coppleson, M., Ed., Gynecologic Oncology, 2nd Edition, Churchill Livingstone, Edinbrugh, 1059-1068.

[6] Ridley, C.M. (2009) The vulva. Neill, S. and Lewis, F. Eds., 3rd Edition, Wiley-Blackwell, Oxford, 211.

[7] de Hullu, J., Hollema, H., Hoeekstra, H.J., Piers, D.A., Mouritis, M.J.E., Aalders, J.G. and van der Zee, A.G.J. (2002) Vulvar melanoma: Is there a role for sentinel lymph node biopsy? Cancer, 94, 486-491. doi:10.1002/cncr.10230

[8] Verschraegen, C.F., Benjapibal, M., Supakarapongkul, W., Levy, L.B., Ross, M., Atkinson, E.N., Bodurka-Bevers, D., Kavanagh, J.J., Kudelka, A.P. and Legha, S.S. (2001) Vulvar melanoma at the M.D. Anderson cancer center: 25 years later. International Journal of Gynecological Cancer, 11, 359-364. doi:10.1046/j.1525-1438.2001.01043.x

[9] Lloyd, J. and Flanagan, A.M. (2000) Mammary and extramammary Paget's disease. Journal of Clinical Pathology, 53, 742-749. doi:10.1136/jcp.53.10.742

[10] Balch, C.M., Gershenwald, J.E., Soong, S., et al. (2009) Final version of 2009 AJCC melanoma staging and classification. Journal of Clinical Oncology, 27, 6199-6206. doi:10.1200/JCO.2009.23.4799

[11] Look, K.Y., Roth, L.M. and Sutton, G.P. (1993) Vulvar melanoma reconsidered. Cancer, 72, 143-146. doi:10.1002/1097-0142(19930701)72:1<143::AID-CNC R2820720127>3.0.CO;2-M

[12] Dunton, C.J., Kautzy, M. and Hanau, C. (1995) Maligant melanoma of the vulva: A review. Obstetrics and Gynecology Survey, 50, 739-746.

[13] de Hullu, J., Oonk, M. and van dar Zee, A. (2004) Modern management of vulvar cancer. Current Opinion in Obstetrics \& Gynecology, 16, 65-72. doi:10.1097/00001703-200402000-00012

[14] Bouma, J., Weening, J.J. and Elders, A. (1982) Malignant melanoma of the vulva: Report of 18 cases. European Journal of Obstetrics \& Gynecology and Reproductive Biology, 13, 237-251. doi:10.1016/0028-2243(82)90105-8

[15] Podratz, K.C., Gaffey, T.A., Symmonds, R.E., et al. (1983) Melanoma of the vulva: An update. Gynecologic Oncology, 16, 153-168. doi:10.1016/0090-8258(83)90090-2

[16] Bradgate, M.J., Rollason, T.P., McConkey, C. and Powell J. (1990) Malignant melanoma of the vulva: A clinico-pathological study of 30 women. British Journal of Obstetrics and Gynaecology, 97, 124-133. doi:10.1111/j.1471-0528.1990.tb01737.x

[17] Miller, B.J., Rutherford, L.F., McLeod, G.R.C. and Cohen, J.R. (2008) Where the sun never shines: Anorectal melanoma. ANZ Journal of Surgery, 67, 846-848. doi:10.1111/j.1445-2197.1997.tb07609.x 